\title{
Redes de Aprendência: uso de tecnologias digitais e formação de professores
}

\author{
Selma Bessa Sales ${ }^{1}$, Silvia Fichmann² \\ ${ }^{1}$ Centro de Referência do Professor (CRP), Núcleo de Tecnologia Educacional (NTE), \\ Secretaria Municipal de Educação de Fortaleza (SME). Rua Conde D’Eu, 560 - Centro, \\ CEP: 60.055-070-Fort/CE. \\ ${ }^{2}$ CETRANS - Centro de Educação Transdisciplinar \\ Rua Claudio Soares, 72 Cj. 809 CEP : 05422 - 030 - SP \\ selmabessa0808@gmail.com, silviafich@gmail.com
}

\begin{abstract}
Abstrat. This article proposes a reflection on the results of the implementation of two training courses developed for school teachers in the promotion of learning networks in Fortaleza city Public Primary Education System. These courses aimed to show possible motivational, dynamic and collaborative ways to integrate digital technologies to the curriculum using virtual environments. The result of this research shows that it is possible to educate the teacher for the creation and promotion of Learning Networks, based on the transdisciplinary foundational elements as an innovative practice regarding an effective use of technologies integrated into the pedagogical project and the use of diversified web.2.0 resources.
\end{abstract}

Resumo. Este artigo propõe uma reflexão sobre os resultados da implementação de dois cursos de formação de professores para a criação e dinamização de Redes de Aprendência na Educação Básica do Sistema Municipal de Ensino de Fortaleza. O objetivo desses cursos foi mostrar caminhos possíveis para a integração de tecnologias digitais aos conteúdos curriculares em ambientes virtuais de forma motivadora, dinâmica $e$ colaborativa. O resultado desta pesquisa demonstrou que é possível formar o professor para a criação e dinamização de Redes de Aprendência, tendo como base a fundamentação transdisciplinar, como uma prática inovadora no que se refere ao uso efetivo de tecnologias integradas ao projeto pedagógico e ao domínio de recursos Web.2.0 diversificados.

\section{Introdução}

Com o advento e evolução das tecnologias digitais da informação e comunicação (TDIC) e o crescente uso na educação, o conhecimento cientifico não é mais imutável, continuamente novas pesquisas derrubam paradigmas estabelecidos e dão lugar a outros. Nesse novo cenário, contamos também com as inúmeras possibilidades oferecidas por essas tecnologias que favorecem a criação de novos espaços de aprendizagem.

Nesse contexto, o quadro que se apresenta na escola, com os professores e alunos, em Fortaleza/CE, não é diferente, os desafios são permanentes. De acordo com Kenski (2003) as mídias invadem o cotidiano, configurando-se como complemento, como companhia, como continuidade do espaço vida e, por meio do que é veiculado por elas, e por meio delas as pessoas se comunicam, adquirem informações e transformam 
seus comportamentos.

Nessa perspectiva, como oferecer ao professor uma formação em que ele possa potencializar o uso das TDIC em sala de aula, uma vez que elas introduzem novos modos de comunicação, permitem a expressão do pensamento pelas modalidades da escrita, da imagem e a combinação dessas modalidades [Almeida, Valente, 2012]. Como formar o professor numa perspectiva de incorporar a tecnologia ao fazer pedagógico, como ser crítico e reflexivo, efetivamente participativo e autor em potencial? Como proporcionar a criação de uma teia dinâmica de troca de informações e experiências para trazer a escola para a rede e a rede da escola para o mundo?

Com a necessidade de um aprimoramento, para aperfeiçoar a forma de se comunicar e de adquirir conhecimentos relacionados ao uso inovador de tecnologias os professores da rede pública de Fortaleza participaram da formação para a integração de ferramentas digitais e criação de redes de aprendência. O processo de desenvolvimento do curso permitiu ao professor refletir e discutir sobre como se apropriar da tecnologia, articular a teoria e a prática e a produção colaborativa de conhecimentos através do uso de ambientes de aprendizagem e recursos digitais como: Fórum Portal do Professor/MEC, redes sociais: Facebook, Twitter, You Tube, Google Drive, suas interfaces de comunicação, e blogs.

A relevância desta pesquisa está relacionada ao uso efetivo das TDIC na escola a partir de redes de aprendência. A investigação tem cunho quanti-qualitativo, a partir da análise dos resultados processuais e de um questionário aplicado, após o curso, aos professores cursistas.

\section{Redes Sociais, Redes de Aprendência e ferramentas digitais colaborativas}

O grande desafio hoje para o professor é a apropriação, no contexto formativo, das ferramentas digitais, seguido do reconhecimento das possibilidades de uso pedagógico. Atualmente as redes sociais são consideradas como espaço virtual de interação que pode se tornar um ambiente favorável ao processo de ensino e aprendizagem, tanto para a formação de professores como de alunos.

As pesquisas relacionadas ao uso de redes sociais na educação, especificamente sobre uso do Facebook, intensificaram-se em 2008, ano em que foram denominadas de "Social Networking Sites" (SNS). Griffith e Liyanage (2008) concluíram que diversas redes e a estrutura social estabelecida nos SNS ajudava a promover a interação entre professor e aluno. Patrício e Gonçalves (2010) desenvolveram um estudo de caso com cinquenta e nove alunos do $1 .^{\circ}$ ano da licenciatura em Educação Básica, na unidade curricular de Informação e Comunicação em Educação, no Instituto Politécnico de Bragança. O estudo consistiu na exploração das aplicações e funcionalidades do Facebook, na identificação da sua utilidade educativa, na experimentação através de recursos e atividades contextualizadas e na avaliação por meio de um questionário. $\mathrm{O}$ resultado desta pesquisa demonstrou que o Facebook pode ser utilizado como recurso pedagógico importante para promover maior participação, interação e colaboração no processo educativo e, pode impulsionar também a construção partilhada, crítica e reflexiva de informação e conhecimento distribuídos em prol da inteligência coletiva.

O professor necessita trabalhar num ambiente criativo, aberto, dinâmico, complexo, como as redes sociais, espaço onde o aluno já se faz presente. A ideia de "redes de aprendência" surge a partir desse desafio e da intenção de apresentar caminhos onde o professor desenvolva projetos transdisciplinares.

Por que redes de aprendência? O termo "aprendência" é um neologismo criado 
por Hélène Trocmé-Fabre, na década de 90, que substitui o termo "aprendizagem", por ser um conceito mais vasto, dinâmico, nômade e mestiço que melhor expressa as pesquisas recentes na área de neurobiologia e, que confirmam que somos capazes de aprender ao longo de toda a vida. Este termo tem sido usado com a finalidade de inovar e ampliar ações educativas, que ocorrem independentemente de tempo e de lugar e por toda a vida. Além disso, Trócme-Fabre (2003) propõe três instrumentos que contribuem para o aprender: questionamento, autoposicionamento e avaliação, que podem ser utilizados nas atividades desenvolvidas nos ambientes virtuais. Estes conceitos e a visão, atitude e práxis transdisciplinar também nortearam este trabalho.

A partir da formação sugerida e com o objetivo de promover ação diante do que foi vivenciado no percurso da formação, de fato, percebeu-se a atuação do professor pertinentes, além da instrumentalização, onde ele torna-se agente, um produtor e crítico das novas tecnologias. O mais significativo nesta experiência foi a percepção que a tecnologia passou a se constituir um meio para o desenvolvimento das ações pedagógicas e a Internet, um ambiente virtual que pode ser integrado à realidade, possibilitando o acesso às informações (Fichmann,2005). Isto é, na promoção da educação online, o importante é a forma como são exploradas as tecnologias, e não o domínio técnico que o professor tem dos recursos.

A Formação constituiu-se, em inspiração para que o docente pudesse incorporar as TDIC à rotina escolar: uma referência para sua prática, algo citado inclusive em revistas especializadas ${ }^{1}$. O professor precisa desenvolver sua competência para depois ajudar os alunos a desenvolverem as suas. Assim, a capacidade de uso dessas tecnologias no desenvolvimento de redes de aprendência passa a ser intimamente relacionada com determinadas competências desenvolvidas pelos professores. Como parte dessa apropriação, é necessário o domínio das ferramentas digitais e saber utilizálas para o desenvolvimento do currículo, para possível elaboração e dinamização das redes de aprendência. Como nos diz Nóvoa (1996) "a inovação só tem sentido se passar por dentro de cada um, se for objeto de reflexão e de apropriação pessoal".

\section{Formação de professores para a criação e dinamização de redes de aprendência}

A formação ocorreu no início de 2013, no Núcleo de Tecnologia Educacional/Fortaleza. Foram ofertadas duas turmas, cada uma com vinte vagas ${ }^{2}$;; capacidade limite do Laboratório de Informática do NTE. Iniciamos o curso com trinta e seis participantes, distribuídos nas duas turmas, e finalizamos com vinte e dois que participaram efetivamente até a finalização da formação

Para alcançar os objetivos propostos, adotou-se como concepção de ensinoaprendizagem a abordagem sócioconstrutivista, na qual cada participante é sujeito de sua própria aprendizagem, destacando o "aprender fazer fazendo", estimulando-se também a aprendizagem colaborativa, aliada às ideias proposta por Trocme-Fabre (2003).

Baseado numa metodologia teórico-prática ${ }^{3}$, o curso foi organizado com carga horária de sessenta horas, em seis momentos presenciais totalizando dezoito horas e

1 Revista ARede- Ano 9/No 89-Março de 2013, P. 13- Disponível em http://arede.inf.br/edicao-n-89marco-2013/5454-capa-comeca-por-voce-professora Acesso 20/03/2013

2 Grade de Cursos NTE/CRP- Disponível em http://www.sme.fortaleza.ce.gov.br/educacao/files/GRADEDEFINITIVA(1).pdf Acesso em 20/01/13

3 Momentos Presenciais- Disponível em: https://sites.google.com/site/aprendencia/sed Acesso 12/02/13 
trinta e duas à distância. O referido curso propôs-se a discutir sobre a utilização das redes sociais e de ferramentas digitais no processo ensino aprendizagem, na perspectiva de rede de aprendência. Partindo da apropriação e uso das TDIC para a elaboração e a dinamização de uma rede colaborativa como suporte para a construção de uma cadeia de conhecimentos de forma significativa promovendo também a incorporação das tecnologias ao cotidiano escolar.O curso culminou com uma ação concreta, na criação de uma Rede de Aprendência.

Para melhor conceituar o tema, e refletir de forma colaborativa foram usados os recursos digitais do Google Drive 4 , para a criação da "Comunidade Redes de Aprendência" foram realizados vários debates online, via hangouts (ferramenta de comunicação síncrona), dentre outros, "Redes sociais numa perspectiva de redes de aprendência" " ${ }^{6}$. Neste espaço de aprendizagem questões referentes à prática docente foram observadas além das estratégias de uso das TDIC. Nesse momento, é importante focar o processo de aprendizagem para além da instrução ou transmissão de conteúdos (Nobre, Nunes, Fivero, Gava e Bazet, 2012, apud Nicolescu,1999).

A formação teve início com a apresentação do Fórum do Portal do Professor ${ }^{7}$, incentivando os cursistas a se cadastrarem e a utilizarem-no como uma rede de aprendência na discussão sobre uso das redes sociais e ferramentas da Web 2.0, norteadas pela transdisciplinaridade. Esse incentivo foi apoiado pela professora Silvia Fichmann, responsável pela mediação do Fórum e teve a tarefa de ser a agente de motivação e orientar os professores na criação de suas Redes em diálogo naquele espaço. Associado a isso, a referida professora-convidada também participou em outros momentos presenciais, via Goolge Hangout, das discussões em que os docentes tiveram a oportunidade de discutir como planejar uma Rede de Aprendência ${ }^{8}$.

$\mathrm{Na}$ tentativa bem sucedida de enriquecer a experiência dos participantes utilizouse também como rede de aprendência, o site da rede social Facebook, a partir do perfil “ Curso Redes Sociais - Redes de Aprendência ${ }^{9}$, que possibilitou aos docentes publicar e refletir sobre postagens no ambiente. Para que as atividades nesses ambientes virtuais funcionassem como Rede de Aprendência foi necessário que as atividades e os projetos fossem relacionados com a realidade dos cursistas a partir de temas relacionados ao que está entre, através e além das disciplinas, incluindo valores e sentimentos e a articulação entre pessoas e áreas de conhecimento, a fim de atender a epistemologia e metodologia transdisciplinar (Nicolescu, 1999).

Outros espaços virtuais foram usados para o curso, destacamos: os aplicativos do

4 O Google Drive é um serviço de disco virtual que permite o armazenamento e compartilhamento de arquivos na nuvem do Google

5 Comunidade Redes de Aprendência - Disponível em; https://plus.google.com/u/0/communities/116474749594813979079 Acesso 14/02/13

6 Redes sociais numa perspectiva de redes de aprendência -Hangouts Disponível em https://www.youtube.com/watch? $=19 \mathrm{~m} 0$ Bvz31g Acesso 05/02/13

7 Uso de redes sociais na escola: criando Redes de Aprendência. Web 2.0 \& TransdisciplinaridadeDisponível em $\underline{\mathrm{http}} / / /$ portaldoprofessor.mec.gov.br/ListarMensagensForum.html?idTopico=122 - Acesso em 29/11/09

8 Hangout gravado no Youtube : Planejando Redes de Aprendência - Disponível em http://www.youtube.com/watch?v=7hGxgcNWaMg Acesso 10/04/13.

9 Facebook Disponível em http://www.facebook.com/RedesdeAprendencia Acesso 27/12/11 
Google Drive ${ }^{10}$, o ambiente virtual de aprendizagem Socrátes ${ }^{11}$, Twitter ${ }^{12}$, Pinterest $t^{13}$ alguns links colaborativos tais como: Rebel Mouse ${ }^{14}$, Scoop.it ${ }^{15}$, a Rede Social Educacional - Redu ${ }^{16}$. E em um ambiente agregador, About.me, foram listados todos os demais criados para o referido curso: Redes de Aprendência ${ }^{17}$.

\section{Apresentando e refletindo sobre a pesquisa e seus resultados}

Trata-se de uma pesquisa descritiva, de abordagem quali-quantitativa, sobre o impacto do Curso de formação sobre Redes de Aprendência na prática pedagógica docente, a partir da vivência de criação de redes de aprendência, bem como, da apropriação de ferramentas digitais no universo escolar.

O objeto de estudo foi o curso "Integração das ferramentas digitais e criação de Redes de Aprendência". O objetivo da investigação ora apresentada é analisar os resultados do curso, do ponto de vista das ações práticas dos professores no contexto de sala de aula. A coleta de dados foi feita a partir da análise das publicações dos ambientes virtuais criados para o curso, dos registros audiovisuais e fotográficos e do questionário aplicado ao final do curso.

\subsection{Procedimentos metodológicos que nortearam o trabalho}

O planejamento didático do curso foi estruturado a partir de um conjunto de competências consideradas relevantes para quem desenvolve atividades com as TDIC. As aulas, bem como a metodologia do curso, foram planejadas e estruturadas com o objetivo de gerar reflexões sobre o uso das ferramentas digitais para a criação de redes de aprendência.

Nesse sentido, cada momento de estudo e interação nos ambientes virtuais de aprendizagem sugeridos, por meio de atividades individuais e coletivas, teve como foco apresentar a possibilidade de transformar o uso das redes sociais e das ferramentas digitais em redes de aprendência por meio de uma metodologia transdisciplinar vivenciada por cada participante.

Para assegurar um bom desempenho e maior eficiência nas atividades de aprendência foi importante adotar algumas rotinas e procedimentos tais como: visitar rotineiramente os recursos digitais utilizados no curso; participar das discussões no Fórum do Portal do Professor (MEC); postar comentários na Comunidade Redes de Aprendência; "compartilhar" e "curtir" as postagens no grupo do Facebook, "seguir" e "participar" em \#Redesdeaprendência no Twitter ${ }^{18}$, participar com comentários no Blog ${ }^{19}$

A avaliação de aprendizagem do curso teve caráter dinâmico e processual, com

10 Apresentação - Google Drive Disponível em https://docs.google.com/presentation/d/11_Rewm1zeINQCuPqzh5tHSjwQZcuRw6sC8KEYX4qqtE/edit\# $\underline{\text { slide }=\text { id.ge } 880 \mathrm{e} 8 \mathrm{e} 7114}$ Acesso em 06/04/13

11 Sistema On line para criação de cursos e projetos - Disponivel em http://www.virtual.ufc.br/socrates Acesso em 12/02/13

12 Twitter - Cursos CRP -Disponível em http://twuitter.com/Bessa_Curso_CRP, Acesso em 20/01/13

13 Pinterest - Disponível em http://www.pinterest.com/aprendencias/ Acesso em 15/02/13

14 Rebel Mouse- Disponível em https://www.rebelmouse.com/RedesdeAprendencia/Acesso 10/03/12

15 Scoop.it- Disponível em http://www.scoop.it/u/curso-redes-sociais\#curatedTopicsTabSelected

Acesso em 21/03/13

16 Redu -Disponível em http://www.redu.com.br/pessoas/redesdeaprendencia/home Acesso em 25/03/13

17 Rede de Aprendência - About-me Disponível em http://about.me/selmabessa0808 Acesso 02/05/13

18 \#RedesdeAprendencia, twitcam.- O Uso Pedagógico do Twitter - Profa. Dra. Renata Aquino Disponível em http://twitcam.livestream.com/dx7b7 Acesso 23/02/13

19 Redes de Aprendência -Disponível em http://redesdeaprendencia.wordpress.com/ Acesso 16/07/2012 
observação do grau de interesse, da participação nas atividades, da qualidade nas interações, seguindo critérios de intervenções que provocavam, estimulavam a cooperação e o crescimento do grupo. Além disso, no sentido de investigar e avaliar o alcance do curso, foi elaborado um questionário, na ferramenta do Google Drive Formulário, que foi enviado para coletar as respostas dos professores ${ }^{20}$. Este mesmo ambiente sistematizou as respostas, a partir de gráficos. Este questionário foi elaborado com tópicos relacionados ao uso das ferramentas digitais, das redes sociais e sua relação com o processo de ensino e aprendizagem para a criação de redes de aprendência. Do universo de trinta e seis professores, vinte e dois responderam, sendo que dezenove concluíram o curso e três desistiram no decorrer do mesmo. A análise dos dados foi realizada por meio da estatística descritiva, onde as variáveis utilizadas para avaliação foram de cunho qualitativo. A seguir, a análise das respostas relevantes.

\subsection{Análise das respostas do questionário}

\subsubsection{Sobre as orientações do curso}

Em relação às orientações sobre o curso, no Gráfico 01 pode-se verificar que todos os professores-cursistas $(100 \%)$ receberam orientações claras sobre a dinamização das redes de aprendência.

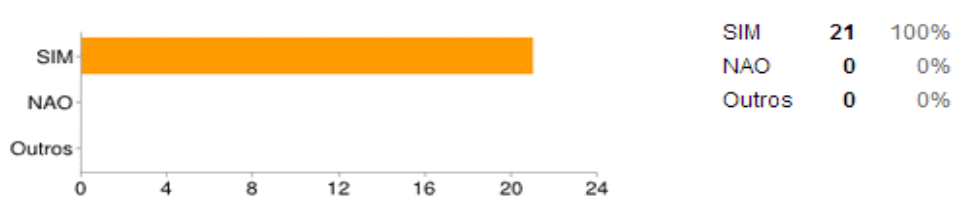

\section{Gráfico 01 - 0 professor recebeu orientações claras durante a Formação}

\subsubsection{Sobre a integração de ferramentas digitais e criação de redes de aprendência}

Os docentes, em sua totalidade, consideraram que é possível desenvolver atividades com os alunos em redes para criar e compartilhar o conhecimento. Aqui em evidência o comentário de uma professora "A formação nos proporcionou uma visão nova das redes de aprendência, descobrimos que podemos aprender de várias maneiras, até mesmo sozinhos"(DO01).

\subsubsection{Sobre a criação de Redes de Aprendência}

Sobre o uso efetivo das Redes de Aprendência, 86\% dos professores desenvolveram atividades extracurriculares utilizando Rede de Aprendência (Gráfico 02), somente um não trabalhou com redes e dois não responderam. Demonstrando que o aprendizado nos momentos presenciais contribuiu para desenvolver as competências e habilidades do professor na apropriação do uso destas Redes. Além disso, 95\% professores respondentes dinamizaram exclusivamente em contextos com propósitos didáticos das disciplinas.
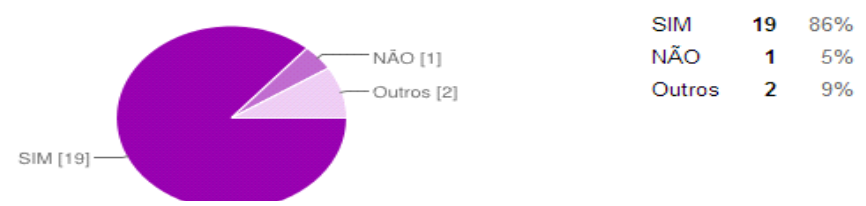

Gráfico 02- Desenvolveram atividades extracurriculares

20 Formulário disponível em https://docs.google.com/forms/d/1MfQbDHEK jv45f8NIPopDRd2I sNLmHCsaVxebXzRhs/edit 


\subsubsection{Sobre as ferramentas digitais utilizadas nas Redes de Aprendência}

Os professores diversificaram o uso de ferramentas digitais para a criação das redes de aprendência. No Gráfico 03 verificou-se que 55\% deles usaram o Blog, 32\% usaram o Facebook, 9\% utilizaram o REDU e apenas um professor usou outra ferramenta.
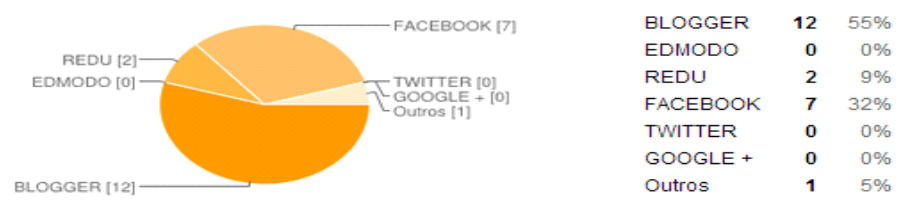

\section{Gráfico 03 - Ferramentas digitais utilizadas para a criação das Redes de Aprendência}

É importante ressaltar que alguns professores fizeram parcerias com seus colegas e criaram as Redes colaborativamente, em pares e que uma das professoras decidiu transferir a sua Rede, que criou em um grupo no Facebook, para um perfil pessoal, pois percebeu que o Grupo não estava dando conta do número de alunos que desejavam participar.

\subsubsection{Sobre a utilização das Redes nos diferentes anos e disciplinas}

Sobre o ano em que a rede de aprendência foi mais utilizada, percebeu-se que a implementação de redes de aprendência teve maior representação no $5^{\circ}$ ano, coincidindo exatamente com o desenvolvimento de ações pedagógicas inseridas, por uma professora-cursista, na Rede de Aprendência elaborada para os Orientadores de Estudos do $5^{\circ}$ ano do Pacto Nacional para Alfabetização na Idade Certa (PAIC e PNAIC) ${ }^{21}$.
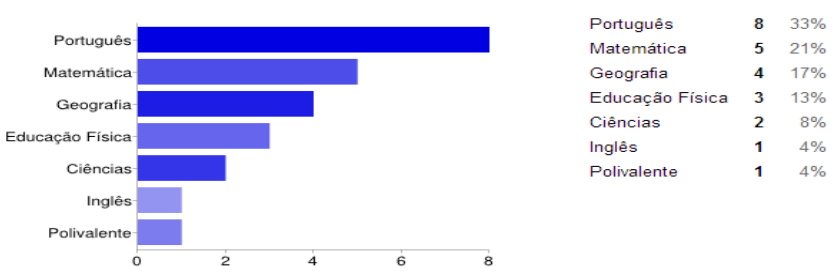

\section{Gráfico 04 -As disciplinas em que as Redes de Aprendência foram utilizadas}

Pode-se perceber, no Gráfico 04 que Português foi a disciplina com mais Redes de Aprendência no contexto da alfabetização e letramento. Por exemplo, a Rede de Aprendência Língua, Literatura e Ciências, Rede de Aprendência Lendo e Re-criando, desenvolvidas na Rede Social Educacional - Redu.

\subsubsection{Sobre a frequência do uso de Redes de aprendência}

A pesquisa indica que $32 \%$ dos professores inseriram duas vezes por semana atividades relacionadas às redes de aprendência na sala de aula, seguido de $23 \%$ de professores que desenvolveram atividades quinzenais. E $27 \%$ dos professores não informaram quantas vezes por semana trabalharam em sala de aula com as Redes. O quadro comprovou que a sala de aula foi um espaço de trabalho com redes de aprendência como prática pedagógica. 

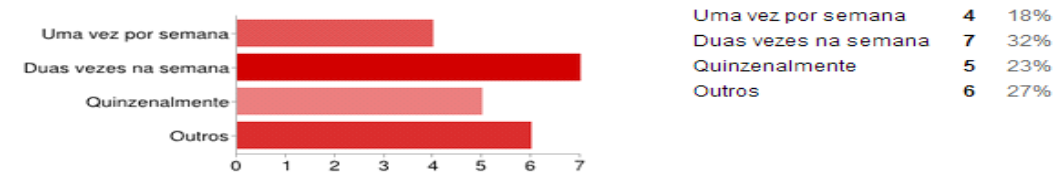

Gráfico 05 - Uso efetivo da Rede de Aprendência na sala de aula

\subsubsection{Sobre o espaço físico onde as atividades aconteceram}

A pesquisa mostrou que $55 \%$ dos docentes coordenaram as atividades de redes de aprendência no Laboratório de Informática - LIE das escolas e 40\% desenvolveram em outros espaços.
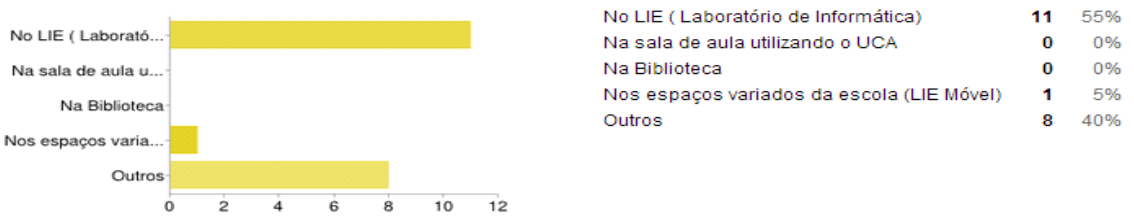

\section{Gráfico 06- Espaços onde as Redes de Aprendência foram dinamizadas}

Os professores demonstraram também interesse em participar de outras formações com foco relacionado a integração das ferramentas digitais da informação e comunicação para a criação de redes de aprendência. Além disso, observou-se em alguns depoimentos dos professores a percepção positiva diante da criação de redes de aprendência:

\footnotetext{
"Deve haver um novo olhar para o uso das Redes Sociais, tendo em vista que muitos profissionais da educação não percebem que estas podem ser ferramentas fundamentais para uma melhor aprendizagem, que vá além dos conteúdos. Os professores podem fazer um melhor uso da tecnologia" (DO2)

"Os alunos destinam tempo de uso das redes para fazer atividades mais produtivas. $^{22}(\mathrm{DO} 3)$
}

\section{As redes de aprendência como inovação na prática pedagógica dos professores e impacto na mudança de comportamento de alunos}

Com base nestes resultados, todos os participantes concordaram que as redes de aprendência são importantes para mobilizar as competências do século XXI e como inspiração da capacidade que os docentes têm para renovar suas práticas de ensino, principalmente no que se refere à integração das tecnologias ao currículo.

Dos professores cursistas participantes, dezenove deles, salientaram que o uso das ferramentas digitais combinados com redes sociais e estratégias pedagógicas bem elaboradas podem inovar a prática pedagógica. A seguir, alguns depoimentos:

\footnotetext{
"Sim, todas as pessoas que estão numa rede social, com a proposta da rede de aprendência, elas compartilham, desenvolvem seus conhecimentos e aprendem numa dinâmica de vários espaços e tempos construindo juntas o conhecimento".(DO4)

"Sim. Pode inovar e transformar totalmente minha prática, pois procurarei pesquisar mais sobre a temática, pensar em utilizar diversas linguagens e fontes de informação e de produção; bem como, a forma como os alunos aprendem e a dinâmica da sala de aula e da escola.(DO5)
}

Nesse estudo, analisamos a participação dos alunos nas redes de aprendência, na

22 http://www.youtube.com/watch?v= xLZ7z12Gak 
promoção de mudança de comportamento. Verificou-se que somente um professor mencionou que não foi possível constatar se o uso das redes de aprendência provocou mudança nos alunos (DO6). Alguns docentes relataram as mudanças observadas em relação ao aprendizado do aluno, o que possibilitou a reflexão sobre a importância do aluno participativo e coautor do processo de aprendizagem.

\footnotetext{
"Ele passou a perceber que podemos utilizar a internet não apenas para interagir nas redes sociais, ouvir música ou assistir vídeos; mas também para ler a notícia, encontrar conteúdos, estudar, opinar, produzir e compartilhar conhecimento".(DO7)

"O interesse pelo uso das ferramentas e principalmente o desenvolvimento da escrita e leitura."(DO8)
}

Observou-se também que, as estratégias utilizadas nas redes de aprendência motivaram os alunos a desenvolver as atividades de casa: "Fez com que os alunos estivessem mais envolvidos com as atividades, interagindo mais e consequentemente aprendendo mais. Elas puderam destinar parte do tempo em casa às atividades da escola. Conseguiram pesquisar e responder no mesmo ambiente virtual."(DO9) ${ }^{23}$

Como balanço da experiência, concluiu-se que os professores foram capazes de desenvolver redes de aprendência para publicação na Web. Todos os dezenove participantes que concluíram a Formação, responderam o questionário, criaram e dinamizaram suas redes de aprendência. Redes de Aprendência elaboradas a partir desse curso: Rede de Aprendência LIE ${ }^{24}$; Redes de Aprendência-Pedagógica ${ }^{25}$; Rede de Aprendência Lendo e Re-criando - Leitura e Produção Textual 50 Ano ${ }^{26}$; Rede de Aprendência Língua, Literatura e Ciências ${ }^{27}$; Rede de Aprendência Alfabetizar Letrando PAIC/PNAIC ${ }^{28}$; EF Profa.Mabelle Aprendência ${ }^{29}$; Redes de Aprendência Geo ${ }^{30}$;Redes De Aprendência Vivenciando a Paz ${ }^{31 ;}$ Matemática na Rede de Aprendência ${ }^{32}$; Rede de Aprendência do Laboratório de Informática ${ }^{33}$; Rede de Aprendência Aluno Eco, é lógico! ${ }^{34}$

\section{Comentários finais}

Essa experiência de formação continuada demonstrou que é possível desenvolver competências no uso de tecnologias digitais a partir do uso de redes de aprendência, com fundamentação e metodologia transdisciplinar. Foi possível a integração de diversos recursos e ambientes online às práticas pedagógicas dos professores, com possibilidade de trabalho em múltiplos espaços: no laboratório de informática, na sala de aula e em ambientes virtuais.

Como reflexão final, percebeu-se também que o professor, diante da inovação vivenciada no processo de Formação, agregou a teoria à prática, através do uso das

23 http://www.youtube.com/watch?v=TYumU dpxRg-

24 https://www.facebook.com/redesdeaprendencia.lie?fref=ts

$25 \mathrm{http}: / /$ redesdeaprendencia.blogspot.com.br

26 http://www.redu.com.br/rede-de-aprendencia-lendo-e-re-criando/cursos/leitura-e-producao-textual-5$\underline{\text { ano }}$

27 http://linguaeliteratura1.blogspot.com.br

28 https://www.facebook.com/kilviaandreia.rededeaprendencia?fref=ts

29 https://www.facebook.com/groups/566470360052716

$30 \mathrm{https}: / / \mathrm{www}$. facebook.com/janaina.geo.9? fref=ts

31 http://redesdeaprendenciavivenciandoapaz.blogspot.com.br/

$32 \mathrm{http}: / /$ matematicanarededeaprendencia.blogspot.com.br/

33 http://rededeaprendenciadolie.blogspot.com.br/2013/04/rede-de-aprendencia-do-laboratorio-de.html

34 http://emeifpadremariano.blogspot.com.br 
TDIC inseridas ao currículo, numa perspectiva além dos conteúdos, nas redes de aprendência. Os participantes demonstraram a necessidade de criar espaços para aprender e ensinar em que docentes e estudantes tornam-se autores, com mediação pedagógica que promove a interação e a produção colaborativa de conhecimento. Talvez a partir destas práticas possamos avançar para uma educação realmente inovadora, que motive e impulsione a aprendência de professores e alunos, em uma escola mais condizente com a realidade atual e com as inúmeras possibilidades que as tecnologias oferecem.

As Redes de Aprendência foram planejadas pelos professores participantes dos cursos e implementadas com seus alunos, no entanto não foi possível avaliar o impacto na aprendizagem. Posteriormente serão feitas pesquisas relacionadas a esse aspecto já que o foco desta pesquisa é o professor e o seu novo fazer pedagógico com o uso de novos recursos disponíveis na Internet.

\section{Referências Bibliográficas}

Almeida, M.E.B, Valente, J. A. Tecnologias e Currículo:Trajetórias convergentes ou divergentes: São Paulo; Paulus, 2012 p. 21 a 26.

Fernandes, L. Redes Sociais Online e Educação: Contributo do Facebook no Contexto das Comunidades Virtuais de Aprendentes. Disponível em http://www.trmef.lfernandes.info/ensaio_TRMEF.pdf_Acesso em 20 set 2013.

Fichmann, S. Formação de Formadores, Transdisciplinaridade e Tecnologia: uma Utopia. In: FRIAÇA, A.; ALONSO, L. K; LACOMBE, M.; BARROS, V. M. Educação e Transdisciplinaridade III. São Paulo: Triom, 2005. p. 415-43

Griffith, S, \& Liyanage, L. (2008). An introduction to the potential of social networking sites in education. In I. Olney, G. Lefoe, J. Mantei, \& J. Herrington (Eds.), Proceedings of the Second Emerging Technologies Conference 2008 (pp.76-81). Wollongong: University of Wollongong.

Kenski , V. M. Tecnologia e Ensino Presencial e a Distância, $4^{\mathrm{a}}$ ed. Campinas, SP: Papirus, 2003.

Mattar, J. O uso de redes sociais na educação-Arquivos do Instituto Ayrton Senna, Entrevista do Portal de Educação e Tecnologia, on line, São Paulo, 2012. Disponível em http://www.educacaoetecnologia.org.br/?p=5487 Acesso em 09 abr 2012.

Nobre.I.AM, Nunes.V.B.,Fávero,R.P, Bazer,L.M.B. Os Processos de Multi, Inter e

Transdisciplinaridade em um Curso voltado para a Formação Continuada de Professores em Informática na Educação - In: Anais do XVIII Workshop de Informática na Escola, 2012. Disponível em: http://www.brie.org/pub/index.php/wie/article/view/2108/1874 Acesso 09 fev 2012.

Nicolescu, B. O Manifesto da Transdisciplinaridade. São Paulo: Triom, 1999.

Nóvoa, A. Relação escola-sociedade: novas respostas para um velho problema. In:

Serbino, Raquel et al. Formação de professores. São Paulo: UNESP. (1996).

Patrício, Maria Raquel; Gonçalves, Vítor (2010) - Facebook: rede social educativa? In I Encontro Internacional TIC e Educação. Lisboa: Universidade de Lisboa, Instituto de Educação. p. 593-598.

Trocmé-Fabre, H. A Árvore do Saber-Aprender. São Paulo: Triom, 2003 